\title{
Radioactive Element
}

National Cancer Institute

\section{Source}

National Cancer Institute. Radioactive Element. NCI Thesaurus. Code C465.

An element where every isotope spontaneously releases radiation until a stable isotope of a different element is generated. 